\title{
DATA EXPERIMENTAL EN LA CALEFACCIÓN SOLAR DE UNA PISCINA CERRADA CON ENVOLVENTE DE POLICARBONATO
}

\section{EXPERIMENTAL DATA ON THE SOLAR HEATING OF A CLOSED POOL WITH POLYCARBONATE COVER}

\section{Cesar Rivasplata-Cabanillas $^{1^{*}(\mathbb{D})}$, Fredy Estalla-Llanque ${ }^{1}{ }^{(\mathbb{B}}$, Edgar Coaquira-Miranda $^{1}$, Victor Arias-Santana ${ }^{2} \oplus$, Sissy Mena-Ordoñez ${ }^{2} \mathbb{0}$}

'Solarsur EIRL, Tacna, Perú.

${ }^{2}$ Universidad Privada de Tacna, Tacna, Perú.

Recibido (Recieved): 21/02/2020 Aceptado (Accepted): 05/03/2020

\begin{abstract}
RESUMEN
La calefacción de piscinas utilizando colectores de placa plana de polipropileno UV, es una práctica conocida en nuestro medio, y tiene justificación técnica y económica en lugares donde existe una excelente radiación solar. El agua de la red es fría (05$15^{\circ} \mathrm{C}$ ), así como las temperaturas ambientes descienden por la noche unos 5 a $10^{\circ} \mathrm{C}$, necesitándose un uso intensivo de la piscina a lo largo de los 12 meses del año, ya sea con fines de recreación, turísticos o terapéuticos. El objetivo de la calefacción es lograr temperaturas de confort en el agua de la piscina, en un rango de $28-32{ }^{\circ} \mathrm{C}$, así como evitar el consumo complementario de energía eléctrica y/o GLP, fundamentalmente a lo largo del periodo estacional más frío del año en el sur del Perú (Abril Agosto), extendiendo así el uso de la piscina hasta casi 12 meses. Una inversión de esta naturaleza, tiene un retorno de capital menor al de 1 año y reditúa largamente en términos técnicos, económicos y de confort. El acoplamiento de un sistema cerrado o envolvente sobre la piscina que genere una atmósfera externa para estadía interna en la piscina, produce un efecto invernadero extremo con temperaturas y humedad en el aire que se encierra en este espacio, que deben ser manejados mediante sistemas de control que activen el movimiento del aire y de la humedad de éste, de modo que se logre temperaturas de confort de cuarto cuando el usuario esta fuera del agua de la piscina. La data experimental adquirida, representa una información valiosa en el análisis térmico de una piscina temperada con colectores solares, con cerramiento volumétrico, lo que permitirá entender el comportamiento térmico de la interacción aire -agua en este tipo de sistemas, y que es diferente al de una piscina temperada a cielo abierto.
\end{abstract}

Palabras claves: colectores de placa plana, envolvente transparente, transferencia de calor, puentes térmicos

\section{ABSTRACT}

The heating of swimming pools using flat plate collectors of polyurethane, is a known practice in our environment, and has technical and economic justification, in places where there is excellent solar radiation, the water of the network is cold (05-15 ${ }^{\circ} \mathrm{C}$ ), as well as the ambient temperatures descend at night about 5 to $10^{\circ} \mathrm{C}$, requiring intensive use of the pool throughout the 12 months of the year, whether for recreational, tourist or therapeutic purposes. The aim of the heating of the pool is to achieve comfort temperatures in the pool water, in a range of $28-32^{\circ} \mathrm{C}$, as well as to avoid the complementary consumption of electrical energy, and / or LPG, mainly along the coldest seasonal period of the year in southern Peru (April - August), thus extending the use of the pool to almost 12 months. An investment of this nature has a return on capital of less than 1 year and results in an investment in technical, economic and comfort terms. The coupling of a closed system on the pool that generates an external atmosphere for internal stay in the pool, produces an extreme greenhouse effect with temperatures and humidity in the air that is enclosed in this space, which must be controlled by control systems that activate the movement of air and humidity of this, so that room comfort temperatures are achieved when the user is out of the pool water. The acquired experimental data represents valuable information in the thermal analysis of a heated pool with solar collectors, with volumetric enclosure, which will allow to understand the thermal behavior of the air-water interaction in this type of systems, and that is different from that of an Outdoor heated pool.

Keywords: flat plate collectors, transparent cover, heat transfer, thermal bridges

\footnotetext{
${ }^{*}$ Corresponding author:

E-mail: cesarrivasplata@yahoo.com
} 


\section{INTRODUCCIÓN}

El calentamiento de agua para piscinas, mediante el uso de colectores solares de placa plana sin cubierta, se justifica, en función al rango de temperaturas de uso $\left(28^{\circ} \mathrm{C}-32^{\circ} \mathrm{C}\right)$, que se requiere por norma y situaciones de confort en la generalidad de las piscinas.

Dependiendo del lugar donde se debe realizar la instalación, y de las condiciones de irradiación promedio $\left(\mathrm{kWh} / \mathrm{m}^{2}\right)$ del lugar, el área de colector necesaria, para cubrir las necesidades de pérdidas de calor en la piscina, serán proporcionales al área de la piscina, en un rango de un $100 \%$ a un $75 \%$ de área del espejo de agua de la piscina.

\section{ANTECEDENTES}

Experiencias sobre calentamiento de piscinas usando colectores solares de placa plana, se vienen dando en Perú, desde hace dos décadas, siendo uno de los primeros desarrollos en el sur del Perú, en Tacna, Moquegua, Toquepala, Cuajone-SPCC-1990 [1] y en el norte en Chiclayo- Aquática [1] [3].

Durante estos últimos años, muchas empresas han incursionado en la instalación de sistemas de calefacción de agua para piscinas, utilizando colectores solares de placa plana importados. (USA, Israel, Brasil) [2]

\section{METODOLOGÍA}

El área del colector es calculada dentro de un balance de energía, que considera por un lado la energía que ingresa al sistema y es capturada por los colectores, y la energía que se pierde diariamente por transferencia de calor a través de la superficie libre de la piscina, la misma que ocurre fundamentalmente, en el caso de una piscina abierta, por procesos convectivos, radiativos, pérdidas por evaporación y pérdidas o adiciones de agua en el proceso de limpieza y filtrado, etc. Las pérdidas de calor consideradas se obtienen a partir de la temperatura de operación de la piscina (usualmente de entre $28^{\circ} \mathrm{C}$ a $30^{\circ} \mathrm{C}$ )[4], [5], [6].

De modo que lo mencionado anteriormente puede ser expresado en una primera aproximación como:

$$
\begin{aligned}
& \eta G \quad A=(P c+P r+P e+P m) \\
& A=(P c+P r+P e+P m) / \eta G
\end{aligned}
$$

Dónde: $\mathrm{G}$ es la irradiación del lugar en $\mathrm{kWh} / \mathrm{m}^{2}$ Pc, Pr, Pe, Pm: Pérdidas por convección, radiación, evaporación y transporte de masa.

$\eta$ : Eficiencia de los colectores.
Para el caso de una piscina cerrada, los fenómenos que se presentan están influenciados por la interacción agua y aire encerrado en el volumen envolvente, y en consecuencia por un intercambio de masa y energía, dentro del volumen encerrado y que influyen en el objetivo final de obtener una temperatura de confort óptima en el agua de la piscina, así como en el aire circundante interior de la piscina.

\subsection{EQUIPOS Y REGISTRO DE DATOS}

En el presente trabajo, para el registro de datos se empleó una placa Arduino Mega 2560, más todos los elementos de control y sensores que permitieron el registro de temperaturas y humedad en el sistema integral de la piscina y su entorno. El equipo en su totalidad fue construido en base a un diseño desarrollado en la empresa y comprado en sus partes, en el mercado local. Los accesorios, materiales y su

\begin{tabular}{|c|c|}
\hline Placa Arduino Mega 2560 & 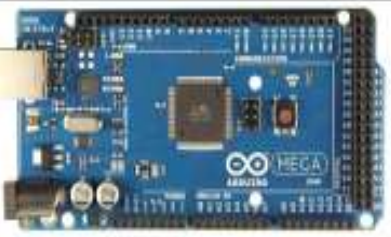 \\
\hline $\begin{array}{l}\text { Módulo RTC DS3231: } \\
\text { Registrar el horario a } \\
\text { tiempo real }\end{array}$ & \\
\hline $\begin{array}{l}\text { Módulo Micro SD } \\
\text { Guardar la información a } \\
\text { una memoria Micro SD } \\
\text { (de acuerdo a los tiempos } \\
\text { establecidos) }\end{array}$ & \\
\hline $\begin{array}{l}\text { Sensor DHT1 } 1 \\
\text { Registrar 1a señales en } \\
\text { basea TEMPERATURA- } \\
\text { HUMEDAD (Ambiente } \\
\text { exterior). }\end{array}$ & \\
\hline $\begin{array}{l}\text { Sensor DHT22 } \\
\text { Registrar la señales on } \\
\text { basea TEMPERATURA- } \\
\text { HUMEDAD (Ambiente } \\
\text { interior }\end{array}$ & \\
\hline $\begin{array}{l}\text { Sensor DS18B20 Para este } \\
\text { caso se usos } 3 \text { sensores de } \\
\text { este modelo para } \\
\Delta \mathrm{T} 1=\text { colectores } \\
\Delta \mathrm{T} \text {. }=\text { Temperatura } \\
\text { superficial de la piscina. } \\
\Delta \mathrm{T} \text {. }=\text { Temperatura a } \\
1 \text { metro de la piscina }\end{array}$ & \\
\hline Pantalla NOKIA 5110 & \\
\hline
\end{tabular}
función se detallan en la fig. 1.

Fig. 1. Funciones de los accesorios del sistema integral de registro de temperatura y humedad del aire en el interior del espacio envolvente de la piscina. 
Este equipo más el sistema central de control diferencial de temperaturas en la piscina, permitieron la obtención de data que se procesó en los gráficos que se muestran en la sección de resultados, a fin de dar una explicación racional de la fenomenología que ocurre en el sistema de calefacción de la piscina. Si bien esta data no es exhaustiva en tiempo es representativa para obtener algunas conclusiones válidas.

\section{ANÁLISIS DE RESULTADOS}

Tal como se observa en las figuras 2 y 3, obtenidas como data experimental, en una piscina temperada ubicada en el distrito de Calana-Tacna (Latitud - $17^{\circ} 57^{\prime}$ $\mathrm{N}$ y Longitud $-70^{\circ} 11^{\prime} \mathrm{E}$ ) existe una marcada correlación entre las temperaturas del aire en exteriores e interiores a la piscina, a lo largo de todo el día, ello como resultado de la cubierta y por la presencia de la masa térmica del agua.

Ha de observarse también, que conforme las temperaturas en exteriores disminuyen, existe un paralelismo con la disminución de las temperaturas en el interior del recinto, lo que es un indicador de pérdidas de calor a través del cobertor de policarbonato a un $\Delta \mathrm{T}$ casi constante, $\mathrm{y}$ a través de los puentes térmicos de la estructura metálica empleada en el cobertor. (Fierro y aluminio)

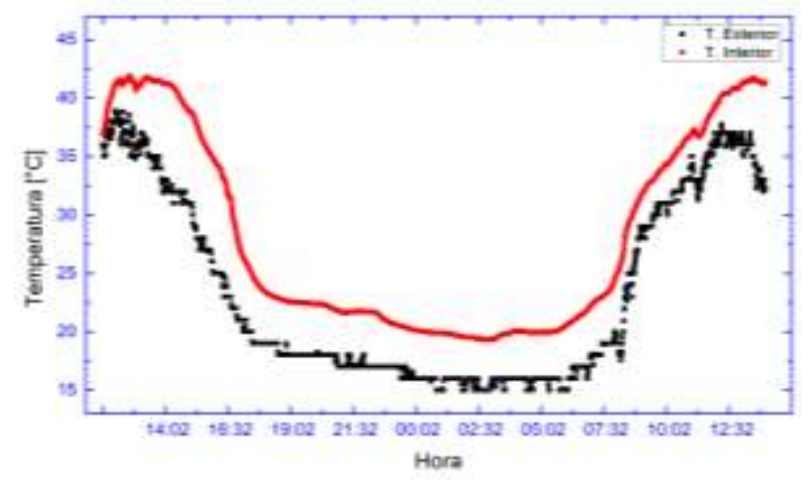

Fig. 2. Temperatura y humedad del aire en el interior del espacio envolvente de la piscina.

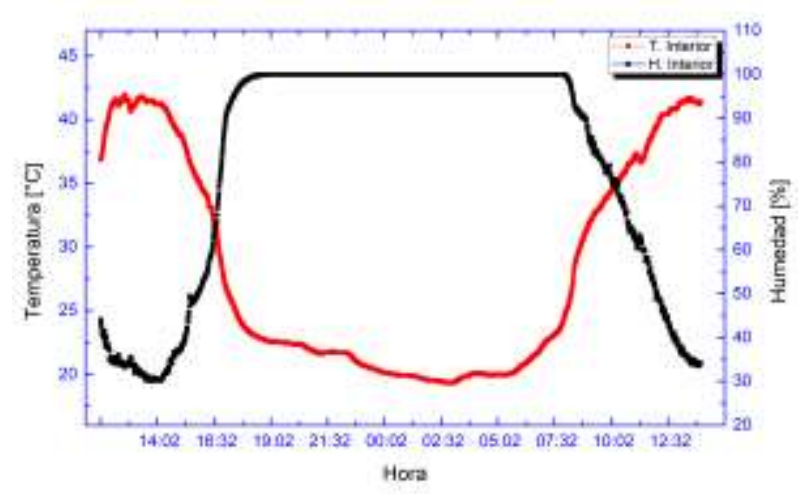

Fig. 3. Perfil de Temperaturas en el agua de la piscina sobre la superficie del agua y a un metro de profundidad.
De la fig. 3, se destaca que la humedad permanece al casi $100 \%$ en un rango de tiempos que van desde las $6 \mathrm{pm}$ a las $7 \mathrm{am}$, destacándose una zona climática comprendida entre $100 \%$ de humedad y temperaturas de $30^{\circ} \mathrm{C}$ a $23^{\circ} \mathrm{C}$ en este mismo rango. Las características de humedad y de temperaturas, definen condiciones ideales para el desarrollo de cultivos bajo invernadero, o para ambientes tipo sauna. El incremento de humedad y el descenso de temperaturas en este espacio de tiempo, permite el traslado de energía por evaporación y transporte de masa de agua, la misma que al condensarse en las paredes del cobertor, no solo hace que haya la necesidad de reponer agua a la piscina, sino también reponer energía durante el día a través de los colectores.

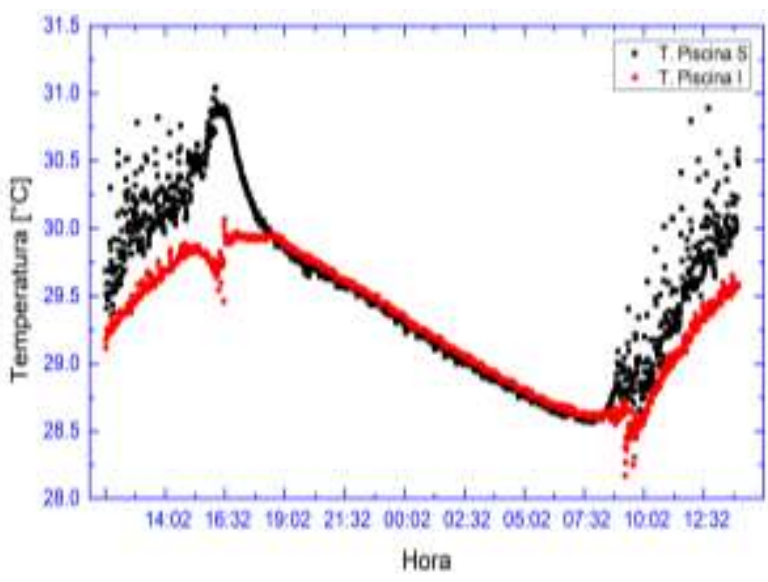

Fig. 4. Perfil de Temperaturas en el agua de la piscina sobre la superficie del agua y a un metro de profundidad.

Al estar la piscina cerrada, se evita en gran medida las pérdidas por convección; sin embargo, la fig. 4 testimonia un descenso sostenido de las temperaturas en el mismo espacio de tiempo que ocurren los procesos de transporte de masa y descenso de las temperaturas en el recinto cerrado, y para el caso del agua en la piscina, desde aproximadamente $30^{\circ} \mathrm{C}$ hasta $28.5^{\circ} \mathrm{C} .\left(1.5^{\circ} \mathrm{C}\right)$.

A fin de lograr una recuperación de todas las pérdidas de calor y lograr la estabilidad de temperaturas de confort en el agua de la piscina, el sistema de calefacción solar empleado, no solo cuenta con un área de colectores del orden de $32 \mathrm{m2}$, que representan casi un $100 \%$ del área de la piscina, sino también con un sistema de control, que comanda el bombeo y circulación del agua de la piscina, a través de los colectores, cada vez que existe una diferencia de temperaturas $\left(\Delta \mathrm{T} \geq 5^{\circ} \mathrm{C}\right.$ ) entre la temperatura de los colectores y la temperatura del agua promedio de la piscina. Ello hasta lograr en la piscina una temperatura de confort del orden de $30^{\circ} \mathrm{C}$. (Fig.5) 


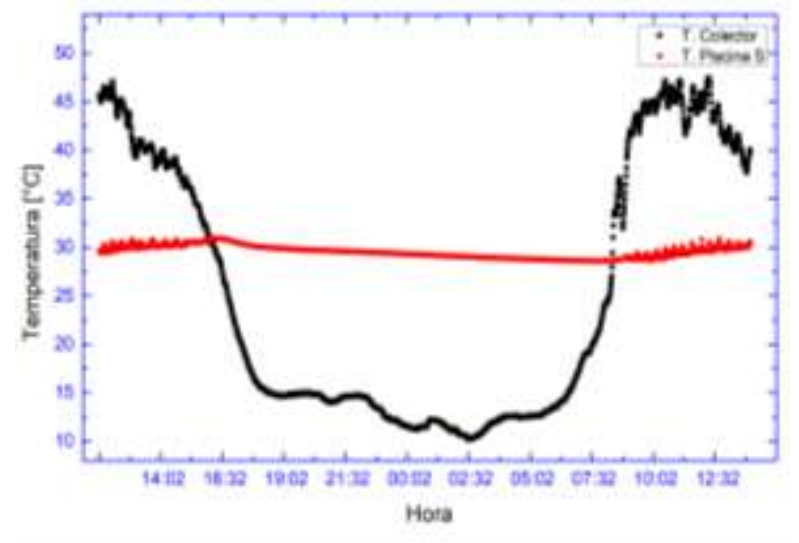

Fig.5. Temperaturas en el colector solar y temperaturas promedio en el agua de la piscina

La Fig. 5, también indica que las temperaturas registradas en el agua de la piscina, cuando se logró la temperatura de confort, tienden a estabilizarse, y a disminuir entre 1 a $2^{\circ} \mathrm{C}$ durante la noche, lo que implica también que, durante el día, la bomba trabajará muy pocas veces, limitándose el consumo de electricidad al mínimo, y con un abastecimiento de energía para la calefacción exclusivamente de origen solar. Toda vez que la presente evaluación se realizó durante estos últimos meses iniciales al verano, es de esperarse, que en los meses más fríos se tenga más pérdidas de calor y un mayor consumo de energía eléctrica.

\section{CONCLUSIONES}

- El calentamiento de agua en una piscina utilizando colectores solares de placa plana, es una práctica usual como una forma de extender el tiempo del verano a una mayor cantidad de meses en el año.

- Técnicamente es posible crear condiciones óptimas en la temperatura del agua de una piscina para un fin determinado, todo ello a través del uso de un sistema central de control programado, mediante el que pueden establecerse los parámetros diferenciales de temperatura, entre la temperatura del colector y el agua de la piscina, y los valores de temperatura máxima y mínima para el agua de la piscina y/o del colector.

- En el caso de las condiciones de climatización logradas en interiores de la piscina, se observa según los resultados, que estos no están en los rangos de confort, ello debido a que el sistema de la piscina, no posee un sistema automático de control que permita la remoción y cambios de aire cuando las temperaturas y la humedad del aire están fuera de esta zona. En parte un cobertor flotante en la superficie de la piscina evitaría ello.

- Así como es factible, lograr piscinas temperadas en climas fríos, también es posible crear condiciones de confort en piscinas donde el clima es extremadamente caluroso y húmedo, o en épocas de verano donde no se requiere mayor rango de temperaturas. El sistema central de control posibilita todo este manejo en la piscina.

- Desde el punto de vista económico, el ahorro de energía que se logra para mantener temperaturas estables en la piscina estudiada, y compensar las pérdidas de calor que ocurren usualmente en una piscina cerrada de $35 \mathrm{~m}^{3}$; para el presente caso los resultados indican, un ahorro equivalente de 45-50 bidones de $10 \mathrm{~kg}$ de GLP/ mes, (valor energético equivalente de $1 \mathrm{~kg}$ de GLP = $14 \mathrm{kWh}$ eléctrico), que a costos actuales significan un gasto de $\mathrm{S} / 1500.00$ soles /mes, y de S/. 18,000.00/año, (\$5,400.00), lo que con una inversión neta de $\$ 4500.00$ para el sistema descrito, se concluye que el tiempo de retorno es ligeramente menor al de un año, siendo altamente rentable.

- Proyecciones de aplicación y de investigación futura nos indican que las piscinas temperadas de uso familiar y/o con fines de uso recreativo, tienen una motivación en su mayoría de casos de tipo terapéutico, y otros, lo que podría crear mejores condiciones y calidad de vida de los usuarios y un uso continuo de la piscina.

- Personas con problemas de la columna y de articulaciones óseas u de otro tipo de problemas de salud relacionados, podrían encontrar mediante el uso de una piscina temperada en varios rangos de temperatura a la usual, un tratamiento hidrotermal adecuado a lo largo de su tratamiento.

- La propuesta ofrece una alternativa positiva como herramienta de confort, siendo una posibilidad altamente confiable en la mejora de calidad de vida, así como en el campo de la medicina de rehabilitación y manejo de estrategias de hidroterapia.

\section{REFERENCIAS}

[1] M. Horn y C. Rivasplata, "Una piscina solar para Toquepala", UNIUNJBG, Tacna, Perú, Inf. téc., 1981.

[2] M. Tinajero y C. Rivasplata, "Sistemas de calentamiento de agua con enegía solar: Una alternativa para el gerenciamiento por el lado de la demanda", en XX Simposio Peruano de Energía Solar y del Ambiente, 11-15 de noviembre, Tacna, Perú, 2013, p. 12.

[3] C. Rivasplata, Aquática, Chiclayo, 1995.

[4] J. T. Czarnecki, "A method of heating swimming pools by solar energy", Sol. Energy, vol. 7, no. 1, pp. 3-7, ene. 1963, doi: 10.1016/0038092X(63)90129-4

[5] J. A. Duffie y W. A. Beckman, Solar Engineering of Thermal Processes., Cuarta edición. United States of America: Wiley, 2013.

[6]J. Twidell y T. Weir, Renewable Energy Resources, Edición: 3. London; New York: Routledge, 2015.

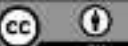

Los artículos publicados por TECNIA pueden ser compartidos a través de la licencia Creative Commons: CC BY 4.0. Permisos lejos de este alcance pueden ser consultados a través del correorevistas@uni.edu.pe 